\title{
Opening Editorial to the Special Issue on the Risk Regulation Reflex
}

In May 2010, the 'Day of Risk' conference was held in The Hague, Netherlands, which later led to the annoucement of the Dutch Risk and Responsibility programme (DRRP). Dr. Adam Burgess, who participated in the conference, suggested to bring some of the material together in a special issue of the EJRR. The publication of this issue comes at a time when the DRRP has delivered some of its preliminary findings at international events, such as SRA Europe's annual conference in June 2012 and the DRRP's own international seminar that took place in The Hague in the same month.

This special issue of the EJRR focuses on the 'risk regulation reflex', a term which originated during the Day of Risk. It has become since then the DRRP's signature term and served as a useful term to trigger debates. Yet its use also needed clarification throughout the time. The DRRP now uses the term to refer to:

- a trend towards ever more far-reaching preventive safety measures which carry the chance of imbalance between the gain in safety and the costs and side effects, and

- the pitfall of a hasty response following an incident leading to disproportionate measures. Disproportionality is a danger not only in legislation but also in regulation, normsetting, implementation and in the responsibility attributed to government.

When such a balance is disturbed, it can hinder society's self-reliance and resilience, restrict the freedom of citizens and businesses, diminish government's authority as a result of that and either stagnate the intended slimming down of government or introduce safety measures which are unnecessarily costly for government or society. This imbalance may be the result of political decisions and can also be enhanced by policy makers or at the level of delivery and local government.

There is an increasing international interest in the problems, dilemmas and undesired effects associated with unbalanced reactions to risk perception.

The articles which make this special issue may help explain some of the problems, and also offer a set of innovative solutions to deal with risk. While four articles were originally written for DRRP and then extended for publication (nrs. 1-3 and 5), three others were especially written for this occasion. All eight contributions offer complementary views on the subject.

The first article, by Margo Trappenburg and Marie-Jeanne Schiffelers, is based on the former's original essay which introduced the term 'risico-regelreflex'. It offers a broad view on the subject, and hones in on the citizen's perspective. The second article, by Michel van Eeten and Frederic Bouder, shows us that politicians tend to act on the assumption that citizens will not accept any risk. It turns out that citizens use a much broader repertoire of moral considerations than just the level of safety. 
Ira Helsloot and Arjen Schmidt take the theme of citizens further when they write about the general public's expectations of government, which seem to be quite different from what government thinks. The article also points out the role of the single-minded safety expert.

Jaap Hanekamp, Jan Kwakman, Roel Pieterman and Paolo Ricci discuss some undesirable effects of the precautionary principle and zero tolerance. Technology for detecting potentially harmful substances is getting to molecular level, where these substances may occur naturally. The authors put forward that overly precautionary food safety regulation may affect food security, which even in the West is much more relevant to human health and life expectancy.

Florentin Blanc focuses on inspections, a field of government intervention which is also prone to the risk regulation reflex. He illustrates how the Soviet system of total control and zero tolerance of risk did not contribute much to safety. Initiatives in former communist countries to introduce a selective risk-based approach form a counterpoint to some Western countries which show tendencies towards more risk-averse regulation.

The UK has been pioneering in the area of disproportionate government response to risks and incidents. The 2006 report 'Risk, Regulation and Responsibility. Whose risk is it anyway?' was highly influential and led to further analysis of mechanisms surrounding public risk. Adam Burgess and Donald Macrae tell us about the origins, effect and legacy of the Risk Regulation Advisory Council (RRAC).

There is one country just across the North Sea which has picked up this legacy. In the final article, I describe what the Dutch Risk and Responsibility programme has done over the past two and a half years and how it is moving towards its conclusion.

In the meantime, there have been some recent developments at EU level which may contribute to a balanced outlook on risk. First of all, the European Food Safety Authority (EFSA) has taken a step towards relaxing the rigidities of zero tolerance by proposing to use the thresholds of toxicological concern for compounds of which no harmful effects are known in small quantities.

A second interesting development is the launch on September 5, 2012 of a new informal Working Group on Risk at the European Parliament. The group will be chaired by Julie Girling MEP (UK Conservative) and involve approximately 10 other MEPs from several political affiliations and member states. Its declared aim is to promote risk based policy making in the EU. The Group will meet three times a year in the European Parliament, assisted by an academic advisory board.

On behalf of the Dutch Risk and Responsibility programme I would like to thank Alberto Alemanno for this welcome opportunity to share and elaborate on some of the insights we have gained, and which may help Dutch 'risk actors' such as politicians and policy makers deal with risks and incidents in a balanced manner. 